\title{
Study of CGMS based Short Duration Hybrids of Pigeonpea (Cajanus cajan (L.) Millsp.) in Terms of Heterosis
}

\author{
J. Srivarsha ${ }^{1}$, J.E. Jahagirdar ${ }^{2}$, C.V. Sameer Kumar ${ }^{*}$, A.J. Hingane ${ }^{1}$, \\ D.K. Patil ${ }^{2}$, V.K. Gite ${ }^{2}$, H.B. Shruthi ${ }^{1}$ and T.M. Bhosle ${ }^{1}$ \\ ${ }^{1}$ International Crops Research Institute for the Semi-Arid Tropics, \\ Patancheru, Hyderabad, Telangana, India \\ ${ }^{2}$ Vasantrao Naik Marathwada Krishi Vidyapeeth, Parbhani, Maharashtra, India \\ *Corresponding author
}

\begin{tabular}{|c|c|}
\hline \multicolumn{2}{|r|}{ A B S T R A C T } \\
\hline & \multirow{6}{*}{$\begin{array}{l}\text { Twenty seven pigeonpea [Cajanus cajan (L.) Millsp.] hybrids were } \\
\text { developed by hand pollination using three CMS lines (A lines) and nine } \\
\text { testers (R lines). These hybrids along with their parents and two standard } \\
\text { checks (VL Arhar1 and ICPL 161) were evaluated in a randomized block } \\
\text { design with three replications during kharif } 2016-17 \text { for the heterosis } \\
\text { studies. Results indicated that the crosses ICPA } 2039 \text { x ICPL161 and ICPA } \\
2039 \text { x ICPL } 90048 \text { had manifested significant heterobeltiosis and standard } \\
\text { heterosis over two checks viz., VL Arhar1 and ICPL } 161 \text { for grain yield per } \\
\text { plant and yield contributing characters. The stability of these promising } \\
\text { crosses can be studied across the different environments and feasibility for } \\
\text { their commercial utilisation could be tested in further generations. }\end{array}$} \\
\hline Keywords & \\
\hline & \\
\hline & \\
\hline Art & \\
\hline & \\
\hline
\end{tabular}

\section{Introduction}

Pigeonpea [Cajanus cajan (L.) Millsp.] $(2 \mathrm{n}=22)$, member of family Leguminosae (Fabaceae) is an important grain legume crop for resource poor farmers of tropics and subtropics. In India, pigeonpea is grown in an area of 5.21 million hectares with a production of 4.23 million tonnes (D.E.S, 2017). Although India leads the world both in area and production of pigeonpea, its productivity is lower $(673 \mathrm{~kg} / \mathrm{ha})$ than the world average $(762.4 \mathrm{~kg} / \mathrm{ha})$ (FAOSTAT, 2015). To promote the pigeonpea production, genetic improvement of pigeonpea was emphasized by researchers for more than five decades and a number of cultivars were developed from hybridization programmes and selection of landraces. However, the progress in the genetic improvement of yield potential has been limited and the improved cultivars failed to enhance the productivity of the crop. Therefore, an alternative breeding approach such as hybrid technology, which has been profitably used in a number of cereals, fruits, and vegetable crops was attempted in pigeonpea to enhance the yield. In 1974, a source of genetic male-sterility 
(GMS) was identified. As a consequence, a GMS based pigeonpea hybrid ICPH 8 was released in 1991 in India (Saxena et al., 1992). Due to the limitation of large-scale hybrid seed production in GMS-based hybrids, the development of cytoplasmic male-sterility (CMS) became imperative.

So far, seven CMS systems have been bred in pigeonpea with varying degrees of success (Saxena and Nadarajan, 2010). Some of the factors responsible for the poor productivity of pigeonpea are the lack of improved cultivars and poor fertility restoration of hybrids.

Research for genetic improvement of this crop, to raise yield levels effectively has to be strengthened countering biotic stresses, through widening genetic base. In view of above consideration, the present study was planned on heterosis and pollen fertility in CGMS-based short duration pigeonpea hybrids.

\section{Materials and Methods}

Three CMS genotypes ICPA 2039, ICPA 2089, ICPA 2156 as lines and nine testers viz., ICPL 88034, ICPL 88039, ICPL 149, ICPL 161, ICPL 81-3, ICPL 89, ICPL 90048, ICPL 86022, ICPL 92047 formed the materials of the present study. They were crossed by hand pollination in a line $\mathrm{x}$ tester design in kharif 2015-16 at International Crops Research Institute for the Semi-Arid Tropics (ICRISAT), Patancheru $\left(17^{0} 53^{1} \mathrm{~N}, 78^{0} 27^{1} \mathrm{E}\right.$, 545.5MSL), India. The resultant twenty seven hybrids along with their parents and standard check varieties (VL Arhar 1 and ICPL 161) were evaluated in a randomized block design with three replications during kharif 2016-17. Each entry was sown in four rows of four metres length with a spacing of $75 \times 30 \mathrm{cms}$ from row to row and plant to plant respectively on $4^{\text {th }}$ of July, 2016.

\section{Data collection}

Five competitive plants of each entry were selected randomly in each replication for recording the observations of eleven characters viz., plant height $(\mathrm{cm})$, days to $50 \%$ flowering, pollen fertility, days to maturity, number of primary branches per plant, number of secondary branches per plant, number of pods per plant, number of seeds per pod, 100 seed weight $(\mathrm{g})$, grain yield per plant $(\mathrm{g})$, harvest index $(\%)$. To record the pollen fertility observation $2 \%$ acetocaramine solution was used. Five well developed flower buds were collected at the time of anthesis from each plant. From each bud, the anthers were collected on a glass slide and crushed with a drop of $2 \%$ acetocaramine stain and examined under a light microscope. The mean value of pollen fertility/sterility of five plants was considered as pollen fertility (\%) for that genotype. The heterosis was calculated as per the procedure suggested by Fonesca and Patterson (1961). The per cent increase or decrease of $F_{1}$ hybrids over better parent as well as standard variety was calculated to estimate possible heterotic effects for above mentioned parameters.

\section{Results and Discussion}

\section{Analysis of variance for line $x$ tester analysis}

In the table 1 the mean sum of squares in the analysis of variance due to lines were significant for all the characters except pollen fertility, number of primary branches per plant, number of seeds per pod, 100 seed weight and harvest index presenting the importance of general combining ability and additive gene effects. The mean sum of squares due to testers were significant for the characters plant height and 100 seed weight showing the importance of general combining ability and additive gene effects. The mean 
sum of squares due to line $\mathrm{x}$ tester were significant for all the characters except number of primary branches per plant, number of secondary branches per plant and number of seeds per pod indicating the impact of specific combining ability and non-additive gene effects.

\section{Estimation of Heterosis}

The success of hybrid breeding depends on the amount of heterosis and the availability of cost-effective hybrid seed production system. In the present investigation, percent heterosis was calculated over better parent (heterobeltiosis) and standard checks viz., VL Arhar1, ICPL 161 (standard heterosis) in twenty seven crosses developed by crossing three lines with nine testers. The magnitude of heterosis varied from trait to trait and cross to cross is presented in the tables 2 and 3.

To achieve high yield, plant height is one of the desirable yield contributing character. For plant height, the heterobeltiosis ranged from 8.33 to 33.50 percent. Eleven crosses exhibited significant positive heterobeltiosis. Out of 27 crosses maximum significant heterobeltiosis is manifested by ICPA $2039 \mathrm{x}$ ICPL 149 (33.50\%) followed by ICPA $2039 x$ ICPL $86022(21.71 \%)$. The range of standard heterosis is -5.44 to 32.72 and -9.53 to 26.98 percent for VL Arhar1 and ICPL 161 respectively. Ten crosses exhibited significant positive heterosis over the check VL Arhar1, of which the cross ICPA 2039 x ICPL 149 $(32.72 \%)$ recorded maximum significant positive heterosis. Five crosses exhibited significant positive heterosis over ICPL 161, of which ICPA 2039 x ICPL 149 (26.98\%) recorded maximum significant positive heterosis. Similar results were also reported earlier by Wankhade et al., (2005), Baskaran and Muthiah(2006), Patel and Tikka, (2008), Sarode et al., (2009), Chandrikala et al., (2010), Vaghela et al., (2011), Pandey et al., (2013), Sudhir et al., (2015).
In context of breeding for short duration hybrids, early flowering and early maturity is generally preferred. So negative heterosis is desirable for flowering and maturity. For days to $50 \%$ flowering, the heterobeltiosis ranges from -12.50 to 14.93 percent. Ten crosses exhibited significant negative heterobeltiosis. Maximum significant negative heterobeltiosis is recorded by ICPA 2089 x ICPL 88034 ($12.50 \%)$. The standard heterosis range is 10 to 33.16 and -17.39 to 0 percent for VL Arhar1 and ICPL 161 respectively. No significant negative heterosis is exhibited over the check VL Arhar1. Out of 27 crosses, 23 crosses manifested significant negative heterosis over the check ICPL 161. Maximum significant negative heterosis is recorded by ICPA 2089 x ICPL $88039(-17.39 \%)$ and ICPA 2156 x ICPL $86022(-17.39 \%)$ over the check ICPL 161.

Heterosis in both negative and positive directions for days to $50 \%$ flowering have also been reported by Wankhade et al., (2005), Baskaran and Muthiah (2006), Wanjari et al., (2007), Patel and Tikka (2008), Sarode et al., (2009), Chandrikala et al., (2010), Vaghela et al., (2011), Pandey et al., (2013).For days to maturity, the range of negative heterobeltiosis is -8.10 to 18.71 percent. Two crosses recorded significant negative heterobeltiosis viz., ICPA 2039 x ICPL $88034(-8.10 \%)$ and ICPA 2089 x ICPL $88034(-7.85 \%)$. The range of standard heterosis ranged from 12.70 to 28.57 and 11.47 to 1 percent for VL Arhar1 and ICPL 161 respectively. None of the crosses exhibited significant negative heterosis over VL Arhar1 for this trait. Out of the twenty seven crosses, the maximum significant negative heterosis was manifested by ICPA 2089 x ICPL $86022(-11.47 \%)$ followed by ICPA 2039 x ICPL 88039 (-9.73\%) over the check ICPL 161. These results are in agreement with earlier results reported by Solanki et al., (2008) and Pandey et al., (2013). 
For the full exploitation of heterosis, hybrids with good amount of fertile pollen are needed. The range of heterobeltiosis for the trait pollen fertility is -13.43 to 0.92 percent. None of the crosses exhibited positive significant heterobeltiosis. The range of standard heterosis is -12.70 to 0.90 and -13.46 to 0.02 for VL Arhar1 and ICPL 161 respectively.

None of the crosses showed significant positive heterosis over all the checks. Results were in agreement with those reported by Wanjari et al., (2007) and Sudhir et al., (2015).

Number of primary branches per plant is one such character which influences productivity. Therefore, the hybrids with more primary branches per plant have to be identified. The range of heterobeltiosis for the trait number of primary branches per plant is -18.26 to 22.50 percent. Two crosses exhibited significant positive heterobeltiosis for this trait viz., ICPA $2039 \times$ ICPL 90048 (22.50\%) and ICPA 2039 x ICPL 81-3 (19.67\%). The range of standard heterosis is -7.96 to 12.89 and 0.78 to 21.69 percent for VL Arhar1 and ICPL 161 respectively.

None of the crosses registered significant negative heterosis over VL Arhar1 for this trait. Over ICPL 161, the cross ICPA $2039 \mathrm{x}$ ICPL 81-3 (21.69\%) showed significant positive heterosis. Similar results were earlier reported by Shoba and Balan (2010), Pandey et al., (2013) and Sudhir et al., (2015).

For the trait number of secondary branches per plant, heterobeltiosis ranged from -13.22 to 20.04 percent. Maximum significant positive heterobeltiosis is exhibited by ICPA 2156 x ICPL 88034 (20.04\%) followed by ICPA 2039 x ICPL 90048 (13.81\%). The range of standard heterosis is -14.31 to 13.64 and -12.63 to 16.55 percent for VL Arhar1 and ICPL 161 respectively. The cross ICPA
2156 x ICPL 88034 (13.64\%) recorded significant positive heterosis over the check VL Arhar1. Three crosses exhibited significant positive heterosis over the check ICPL 161. Maximum significant positive heterosis was shown by ICPA $2156 \mathrm{x}$ ICPL $88034(16.55 \%)$ followed by ICPA $2039 \times$ ICPL 90048 (14.59\%) over the check ICPL 161. Results were in conformity with those obtained by Wankhade et al., (2005), Baskaran and Muthiah (2006), Patel and Tikka (2008), Sarode et al., (2009), Chandrikala et al., (2010), Vaghela et al., (2011), Pandey et al., (2013) and Sudhir et al., (2015).

More number of pods per plant are believed to be closely related to achieve high yield. The range of heterobeltiosis for the trait number of pods per plant is -51.09 to 64.68 percent. Out of twenty seven crosses, nine crosses manifested significant positive heterobeltiosis.

Maximum significant positive heterobeltiosis is exhibited by ICPA 2039 x ICPL 90048 $(64.68 \%)$ followed by ICPA $2039 \times$ ICPL $88034(45.37 \%)$. The range of standard heterosis was -38.49 to 126.33 and -62.83 to 36.76 percent for VL Arhar1 and ICPL 161 respectively. Maximum significant positive heterosis was recorded by ICPA 2039 x ICPL 161 over both the checks VL Arhar1 and ICPL 161. These results are in agreement with the finding of Baskaran and Muthiah (2006), Patel and Tikka (2008), Sarode et al., (2009), Chandrikala et al., (2010), Vaghela et al., (2011), Pandey et al., (2013) and Sudhir et al., (2015).

Positive heterosis for number of seeds per pod is found to be desirable to increase the yield. For the trait number of seeds per pod, heterobeltiosis ranged from -19.23 to 7.14 percent. None of the crosses exhibited significant positive heterobeltiosis. 
Table.1 ANOVA for L x T analysis

\begin{tabular}{|c|c|c|c|c|c|c|c|c|c|c|c|c|}
\hline \multirow[b]{2}{*}{$\begin{array}{l}\text { Sources of } \\
\text { variation }\end{array}$} & \multirow[b]{2}{*}{ d. f. } & \multicolumn{11}{|c|}{ Mean sum of squares } \\
\hline & & $\begin{array}{c}\text { Plant } \\
\text { height } \\
(\mathbf{c m})\end{array}$ & $\begin{array}{c}\text { Days to } 50 \\
\% \\
\text { flowering }\end{array}$ & $\begin{array}{l}\text { Pollen } \\
\text { fertility } \\
(\%)\end{array}$ & $\begin{array}{l}\text { Days to } \\
\text { maturity }\end{array}$ & $\begin{array}{c}\text { Number of } \\
\text { primary } \\
\text { branches } \\
\text { per plant }\end{array}$ & $\begin{array}{c}\text { No. of } \\
\text { secondary } \\
\text { branches } \\
\text { per plant }\end{array}$ & $\begin{array}{c}\text { Number of } \\
\text { pods per plant }\end{array}$ & $\begin{array}{c}\text { No. of } \\
\text { seeds } \\
\text { per } \\
\text { pod }\end{array}$ & $\begin{array}{c}100 \\
\text { seed } \\
\text { weight } \\
(\mathrm{g})\end{array}$ & $\begin{array}{l}\text { Grain } \\
\text { yield per } \\
\text { plant (g) }\end{array}$ & $\begin{array}{c}\text { Harvest } \\
\text { Index }(\%)\end{array}$ \\
\hline Replications & 2 & 72.99 & 15.46 & 2.41 & 24.98 & 0.60 & 4.82 & 301.46 & 0.06 & 0.16 & 11.08 & 1.56 \\
\hline Crosses & 26 & $525.90 * *$ & $57.12 * *$ & $65.41 * *$ & $60.61 * *$ & 0.79 & $7.25 * *$ & $17538.31 * *$ & 0.07 & $0.81 * *$ & $1518.42 * *$ & $117.19 * *$ \\
\hline Parents (Line) & 2 & $2552.01 * *$ & $394.16 * *$ & 168.10 & $202.31 *$ & 1.27 & $25.76^{*}$ & $100271.93 * *$ & 0.02 & 0.88 & $7695.10 * *$ & 189.37 \\
\hline Line $\mathrm{x}$ Tester & 16 & $184.05 * *$ & $20.98 * *$ & $65.19 * *$ & $50.28 * *$ & 0.58 & 4.61 & $8996.00 * *$ & 0.06 & $0.52 *$ & $1017.57 * *$ & $125.65 * *$ \\
\hline Error & 52 & 45.03 & 6.53 & 6.04 & 18.19 & 0.83 & 2.69 & 142.91 & 0.05 & 0.24 & 29.74 & 15.97 \\
\hline
\end{tabular}

*- Significant at $5 \%$ level of significance, $* *$ - Significant at $1 \%$ level of significance

Note: A lines and B lines are isogenic except for pollen fertility. The observations of yield and yield contributing characters except pollen fertility were recorded on B-lines (ICPB 2039, ICPB 2089 and ICPB 2156).

Table.2 Estimation of heterobeltiosis for yield and yield contributing characters

\begin{tabular}{|c|c|c|c|c|c|c|c|c|c|c|c|c|}
\hline $\begin{array}{l}\text { Sr. } \\
\text { No }\end{array}$ & Crosses & $\begin{array}{l}\text { Plant height } \\
\quad \text { (cm) }\end{array}$ & $\begin{array}{c}\text { Days to } 50 \\
\text { per cent } \\
\text { flowering }\end{array}$ & $\begin{array}{l}\text { Pollen } \\
\text { fertility } \\
(\%)\end{array}$ & $\begin{array}{l}\text { Days to } \\
\text { maturity }\end{array}$ & $\begin{array}{c}\text { No. of } \\
\text { primary } \\
\text { branches per } \\
\text { plant }\end{array}$ & $\begin{array}{c}\text { No. of } \\
\text { secondary } \\
\text { branches per } \\
\text { plant }\end{array}$ & $\begin{array}{l}\text { No. of pods } \\
\text { per plant }\end{array}$ & $\begin{array}{l}\text { No. of seeds } \\
\text { per pod }\end{array}$ & $\begin{array}{l}100 \text { seed } \\
\text { wt. (g) }\end{array}$ & $\begin{array}{l}\text { Grain yield } \\
\text { Per Plant } \\
\text { (g) }\end{array}$ & $\begin{array}{c}\text { Harvest } \\
\text { Index } \\
(\%)\end{array}$ \\
\hline 1. & ICPA 2039 x ICPL 88034 & $12.87^{* *}$ & $-6.85^{* *}$ & -2.34 & $-8.10^{* *}$ & $-16.80^{* *}$ & 6.15 & $45.37^{* *}$ & 1.79 & 0.41 & $40.84^{* *}$ & $-20.16^{*}$ \\
\hline 2. & ICPA 2039 x ICPL 88039 & $9.87^{*}$ & $-5.63^{*}$ & 0.92 & -5.07 & -2.59 & 11.91 & -5.93 & 1.79 & $-18.64^{* *}$ & -9.48 & 0.05 \\
\hline 3. & ICPA 2039 x ICPL 149 & $33.50^{* *}$ & $5.44^{*}$ & 0.10 & $8.00^{* *}$ & 10.14 & 5.88 & $12.02^{* *}$ & 0.00 & 4.60 & $21.10^{* *}$ & 3.40 \\
\hline 4. & ICPA 2039 x ICPL 161 & $12.50^{* *}$ & 2.47 & $-5.07^{* *}$ & 4.21 & 5.44 & 11.59 & $30.61^{* *}$ & 0.89 & 2.51 & $32.76^{* *}$ & $-15.86^{*}$ \\
\hline 5. & ICPA 2039 x ICPL $81-3$ & $14.93^{* *}$ & $7.20^{* *}$ & -0.47 & $6.38^{*}$ & $19.67^{*}$ & 8.48 & $-12.70^{* * 3}$ & -1.77 & 5.86 & -9.46 & $-22.93^{* *}$ \\
\hline 6. & ICPA 2039 x ICPL 89 & $18.86^{* *}$ & 3.90 & $-13.43^{* *}$ & 3.73 & 6.55 & 11.15 & $-12.56^{* *}$ & 0.00 & -2.51 & $-21.99^{* * *}$ & $-36.33^{* *}$ \\
\hline 7. & ICPA 2039 x ICPL 90048 & $12.49^{* *}$ & $7.79^{* *}$ & $-3.54^{*}$ & $6.93^{*}$ & $22.50^{* *}$ & $13.81^{*}$ & $64.68^{* *}$ & -6.67 & 4.63 & $83.25^{* *}$ & -7.14 \\
\hline 8. & ICPA 2039 x ICPL 86022 & $21.71^{* *}$ & -1.73 & -2.21 & 1.33 & 6.68 & 4.63 & $-14.15^{* *}$ & 0.88 & -2.89 & $-24.87^{* *}$ & 16.86 \\
\hline 9. & ICPA 2039 x ICPL 92047 & $18.20^{* *}$ & 3.00 & -0.53 & 0.00 & -3.53 & -7.75 & $36.07^{* * *}$ & 0.89 & $-10.89^{*}$ & $21.47^{* *}$ & $-27.93^{* *}$ \\
\hline 10. & ICPA 2089 x ICPL 88034 & 3.47 & $-12.50^{* *}$ & $-4.02^{*}$ & $-7.85^{* *}$ & $-18.26^{* *}$ & 10.37 & $21.50^{* *}$ & $-19.23^{* *}$ & 2.89 & $17.18^{*}$ & -4.42 \\
\hline 11. & ICPA $2089 \times$ ICPL 88039 & -0.35 & 2.45 & $-3.66^{*}$ & $6.47^{*}$ & -8.18 & 0.59 & -8.07 & -4.62 & $-17.63^{* *}$ & $-14.69^{*}$ & $36.99^{* * *}$ \\
\hline 12. & ICPA 2089 x ICPL 149 & $19.80^{* *}$ & $-5.44^{*}$ & $-4.43^{*}$ & -3.47 & -5.80 & 8.38 & $-21.93^{* *}$ & $-7.69^{*}$ & -1.77 & $-25.44^{* *}$ & 9.35 \\
\hline 13. & ICPA 2089 x ICPL 161 & $-8.33^{*}$ & $-5.76^{*}$ & $-13.13^{* *}$ & 1.05 & -5.86 & $13.32^{*}$ & $-40.73^{* *}$ & $-13.08^{* *}$ & -8.40 & $-43.25^{* *}$ & -9.30 \\
\hline 14. & ICPA 2089 x ICPL 81-3 & -0.68 & $-6.36^{*}$ & $-12.94^{* *}$ & -1.60 & -5.92 & 10.81 & $-15.40^{* *}$ & $-12.31^{* *}$ & 4.27 & -9.05 & -11.37 \\
\hline 15. & ICPA 2089 x ICPL 89 & -1.27 & 2.33 & $-10.98^{* *}$ & 3.98 & 0.63 & 7.29 & $21.48^{* *}$ & $-13.08^{* *}$ & 6.84 & $39.66^{* *}$ & 8.92 \\
\hline 16. & ICPA 2089 x ICPL 90048 & 6.85 & -0.46 & $-5.28^{* *}$ & 4.10 & -7.34 & 5.12 & 0.62 & $-11.54^{* *}$ & 3.86 & -5.97 & -4.40 \\
\hline 17. & ICPA $2089 \times$ ICPL 86022 & 3.55 & $5.88^{*}$ & -1.27 & 4.41 & -5.77 & -6.08 & $-23.96^{* *}$ & $-14.62^{* *}$ & 1.65 & -6.67 & -0.24 \\
\hline
\end{tabular}

* - Significant at $5 \%$ level of significance, ** - Significant at $1 \%$ level of significance 
Table.2 Continued.....

\begin{tabular}{|c|c|c|c|c|c|c|c|c|c|c|c|c|}
\hline $\begin{array}{l}\text { Sr. } \\
\text { No }\end{array}$ & Crosses & $\begin{array}{c}\text { Plant } \\
\text { height } \\
(\mathbf{c m})\end{array}$ & $\begin{array}{c}\text { Days to 50 } \\
\text { per cent } \\
\text { flowering } \\
\end{array}$ & $\begin{array}{c}\text { Pollen } \\
\text { fertility } \\
(\%)\end{array}$ & $\begin{array}{l}\text { Days to } \\
\text { maturity }\end{array}$ & $\begin{array}{c}\text { No. of primary } \\
\text { branches per } \\
\text { plant }\end{array}$ & $\begin{array}{c}\text { No. of secondary } \\
\text { branches per } \\
\text { plant }\end{array}$ & $\begin{array}{c}\begin{array}{c}\text { No. of } \\
\text { pods per } \\
\text { plant }\end{array} \\
\end{array}$ & $\begin{array}{l}\text { No. of seeds } \\
\text { per pod }\end{array}$ & $\begin{array}{c}100 \text { seed wt. } \\
\text { (g) }\end{array}$ & $\begin{array}{c}\text { Seed yield } \\
\text { Per Plant (g) }\end{array}$ & $\begin{array}{c}\text { Harvest } \\
\text { Index } \\
(\%) \\
\end{array}$ \\
\hline 18. & ICPA 2089 x ICPL 92047 & -5.91 & $-6.44^{*}$ & $-12.92^{* *}$ & 0.00 & -7.18 & -10.08 & -7.40 & $-13.08^{* *}$ & 2.82 & -12.57 & 2.27 \\
\hline 19. & ICPA 2156 x ICPL 88034 & $12.62^{* *}$ & $-8.87^{*}$ & $-6.26^{* *}$ & -4.81 & $-16.61^{* *}$ & $20.04^{* *}$ & $-12.59^{*}$ & 0.89 & 2.48 & -14.72 & $-24.10^{* * *}$ \\
\hline 20. & ICPA 2156 x ICPL 88039 & -0.25 & $14.93^{* *}$ & $-12.44^{* *}$ & $18.71^{* *}$ & -4.69 & -8.22 & $-16.34^{* *}$ & 7.14 & $-8.47^{*}$ & $-18.96^{* *}$ & 3.31 \\
\hline 21. & ICPA 2156 x ICPL 149 & 4.89 & -4.18 & -0.03 & 2.13 & -3.08 & -9.01 & $-32.05^{* *}$ & 0.00 & 5.70 & $-32.54^{* *}$ & 14.50 \\
\hline 22. & ICPA 2156 x ICPL 161 & -3.95 & $-6.58^{* *}$ & -0.37 & -1.58 & 5.03 & 5.71 & $-51.09^{* *}$ & 0.00 & -3.36 & $-55.53^{* *}$ & -1.91 \\
\hline 23. & ICPA 2156 x ICPL $81-3$ & 2.94 & -3.39 & -2.88 & -1.06 & 0.33 & 2.25 & $-44.03^{* *}$ & 0.88 & $-14.10^{* *}$ & $-35.31^{* *}$ & -0.57 \\
\hline 24. & ICPA 2156 x ICPL 89 & 0.75 & -1.40 & 0.05 & 5.11 & 5.96 & -0.17 & 9.81 & 2.68 & $-16.24^{* *}$ & $21.55^{* *}$ & $-40.52^{* *}$ \\
\hline 25. & ICPA 2156 x ICPL 90048 & 3.24 & -2.30 & $-4.75^{* *}$ & 0.82 & 8.01 & $-13.22^{*}$ & $16.34^{* *}$ & 5.00 & 4.25 & 0.63 & $23.36^{* *}$ \\
\hline 26. & ICPA 2156 x ICPL 86022 & 7.23 & 3.98 & -1.17 & $13.25^{* *}$ & -1.69 & -10.39 & $25.63^{* *}$ & -4.42 & 0.83 & $47.50^{* *}$ & -8.44 \\
\hline \multirow[t]{4}{*}{27.} & ICPA 2156 x ICPL 92047 & 6.05 & $-7.30^{* *}$ & $-5.44^{* *}$ & -2.63 & -5.71 & -9.61 & -3.48 & -4.46 & -4.84 & 0.40 & 14.85 \\
\hline & $\mathrm{SE}(\mathrm{d}) \pm$ & 5.49 & 1.92 & 1.67 & 3.61 & 0.76 & 1.21 & 9.58 & 0.16 & 0.37 & 4.53 & 3.15 \\
\hline & $\mathrm{CD}$ at $5 \%$ & 11.01 & 3.84 & 3.35 & 7.25 & 1.51 & 2.42 & 19.22 & 0.32 & 0.74 & 9.08 & 6.31 \\
\hline & $\mathrm{CD}$ at $1 \%$ & 14.67 & 5.12 & 4.46 & 9.66 & 2.02 & 3.22 & 25.6 & 0.43 & 0.98 & 12.1 & 8.41 \\
\hline
\end{tabular}

* - Significant at $5 \%$ level of significance, ** - Significant at $1 \%$ level of significance

Table.3 Estimation of standard heterosis over the checks VL Arhar1 (Standard Check 1) and ICPL 161 (Standard Check 2)

\begin{tabular}{|c|c|c|c|c|c|c|c|c|c|c|c|c|c|}
\hline \multirow[t]{2}{*}{$\begin{array}{l}\text { Sr. } \\
\text { No }\end{array}$} & \multirow[t]{2}{*}{ Crosses } & \multicolumn{2}{|c|}{ Plant height (cm) } & \multicolumn{2}{|c|}{$\begin{array}{c}\text { Days to } 50 \text { per cent } \\
\text { flowering }\end{array}$} & \multicolumn{2}{|c|}{$\begin{array}{c}\text { Pollen fertility } \\
(\%)\end{array}$} & \multicolumn{2}{|c|}{ Days to maturity } & \multicolumn{2}{|c|}{$\begin{array}{c}\text { No. of primary } \\
\text { branches per plant }\end{array}$} & \multicolumn{2}{|c|}{$\begin{array}{c}\text { No. of secondary branches } \\
\text { per plant }\end{array}$} \\
\hline & & SC 1 & SC 2 & SC 1 & SC 2 & SC 1 & SC 2 & SC 1 & SC 2 & SC 1 & SC 2 & SC 1 & SC 2 \\
\hline 1. & ICPA 2039 x ICPL 88034 & $10.84 * *$ & 6.05 & $21.58 * *$ & $-8.70 * *$ & -1.19 & -2.05 & $15.24 * *$ & $-9.48 * *$ & -5.03 & 2.37 & 0.50 & 3.07 \\
\hline 2. & ICPA 2039 x ICPL 88039 & 5.49 & 0.93 & $14.74 * *$ & $-13.83^{* *}$ & 0.78 & -0.10 & $13.02 * *$ & $-11.22 * *$ & -5.35 & 2.03 & 10.98 & $13.82 *$ \\
\hline 3. & ICPA 2039 x ICPL 149 & $32.72 * *$ & $26.98^{* *}$ & $32.63 * *$ & -0.40 & 0.14 & -0.74 & $28.57 * *$ & 1.00 & -4.40 & 3.05 & -1.16 & 1.37 \\
\hline 4. & ICPA 2039 x ICPL 161 & $24.70^{* *}$ & $19.30^{* *}$ & $31.05^{* *}$ & -1.58 & $-4.30 *$ & $-5.14 * *$ & $25.71 * *$ & -1.25 & -2.52 & 5.08 & 7.32 & 10.07 \\
\hline 5. & ICPA 2039 x ICPL 81-3 & $23.48 * *$ & $18.14^{* *}$ & $33.16^{* *}$ & 0.00 & 0.58 & -0.30 & $26.98 * *$ & -0.25 & 12.89 & $21.69 * *$ & 4.33 & 7.00 \\
\hline 6. & ICPA 2039 x ICPL 89 & $9.38^{*}$ & 4.65 & $26.32 * *$ & $-5.14 *$ & $-12.70^{* * *}$ & $-13.46^{* *}$ & 23.49 ** & -2.99 & -2.83 & 4.75 & 7.82 & 10.58 \\
\hline 7. & ICPA $2039 \times$ ICPL 90048 & 6.37 & 1.77 & $31.05 * *$ & -1.58 & -2.27 & -3.12 & $27.30^{* * *}$ & 0.00 & 6.32 & 14.61 & 11.73 & $14.59^{*}$ \\
\hline 8. & ICPA 2039 x ICPL 86022 & 3.55 & -0.93 & $19.47 * *$ & $-10.28 * *$ & -1.05 & -1.91 & $20.63 * *$ & -5.24 & -7.08 & 0.17 & 0.83 & 3.41 \\
\hline 9. & ICPA 2039 x ICPL 92047 & $21.54 * *$ & $16.28^{* *}$ & $26.32 * *$ & $-5.14 *$ & 0.88 & 0.00 & $20.63 * *$ & -5.24 & -5.35 & 2.03 & -1.00 & 1.54 \\
\hline 10. & ICPA 2089 x ICPL 88034 & 1.60 & -2.79 & $14.21 * *$ & $-14.23 * *$ & -2.88 & $-3.73^{*}$ & $15.56^{* *}$ & $-9.23 * *$ & -6.70 & 0.58 & 4.49 & 7.17 \\
\hline 11. & ICPA 2089 x ICPL 88039 & -4.33 & $-8.47 *$ & $10.00 * *$ & $-17.39 * *$ & $-3.79 *$ & $-4.63^{* *}$ & $14.92 * *$ & $-9.73 * *$ & -7.89 & -0.71 & -0.25 & 2.30 \\
\hline 12. & ICPA 2089 x ICPL 149 & $19.11 * *$ & $13.95 * *$ & $18.95 * *$ & $-10.67 * *$ & $-4.40 *$ & $-5.24 * *$ & $14.92 * *$ & $-9.73 * *$ & -5.50 & 1.86 & 1.16 & 3.75 \\
\hline 13. & ICPA 2089 x ICPL 161 & 1.60 & -2.79 & $20.53^{* *}$ & $-9.49 * *$ & $-12.43 * *$ & $-13.19 * *$ & $21.90 * *$ & -4.24 & -5.57 & 1.80 & 8.99 & 11.77 \\
\hline 14. & ICPA $2089 \times$ ICPL $81-3$ & 6.71 & 2.09 & $16.32 * *$ & $-12.65 * *$ & $-12.02 * *$ & $-12.79 * *$ & $17.46^{* * *}$ & $-7.73 * *$ & -5.63 & 1.73 & 6.57 & 9.30 \\
\hline 15. & ICPA 2089 x ICPL 89 & -5.44 & $-9.53^{*}$ & $15.79 * *$ & $-13.04 * *$ & $-10.23^{* * *}$ & $-11.01 * *$ & $16.19^{* *}$ & $-8.73 * *$ & 0.94 & 8.81 & 4.08 & 6.74 \\
\hline 16. & ICPA $2089 \times$ ICPL 90048 & 2.33 & -2.09 & $13.68^{* *}$ & $-14.62 * *$ & $-4.03 *$ & $-4.87 * *$ & $20.95 * *$ & -4.99 & -7.04 & 0.20 & 3.19 & 5.84 \\
\hline 17. & ICPA 2089 x ICPL 86022 & -0.83 & -5.12 & $13.68 * *$ & $-14.62 * *$ & -0.10 & -0.97 & $12.70^{* *}$ & $-11.47 * *$ & -5.47 & 1.90 & -9.48 & -7.17 \\
\hline
\end{tabular}

* - Significant at $5 \%$ level of significance, ${ }^{* *}$ - Significant at $1 \%$ level of significance 
Table.3 Continued.....

\begin{tabular}{|c|c|c|c|c|c|c|c|c|c|c|c|c|c|}
\hline $\begin{array}{l}\text { Sr. } \\
\text { No }\end{array}$ & Crosses & \multicolumn{2}{|c|}{ Plant height (cm) } & \multicolumn{2}{|c|}{$\begin{array}{l}\text { Days to } 50 \text { per cent } \\
\text { flowering }\end{array}$} & \multicolumn{2}{|c|}{$\begin{array}{c}\text { Pollen fertility } \\
(\%)\end{array}$} & \multicolumn{2}{|c|}{ Days to maturity } & \multicolumn{2}{|c|}{$\begin{array}{c}\text { No. of primary branches } \\
\text { per plant }\end{array}$} & \multicolumn{2}{|c|}{$\begin{array}{c}\text { No. of secondary branche } \\
\text { per plant }\end{array}$} \\
\hline & & SC 1 & SC 2 & SC 1 & SC 2 & SC 1 & SC 2 & SC 1 & SC 2 & SC 1 & SC 2 & SC 1 & SC 2 \\
\hline 18. & ICPA 2089 x ICPL 92047 & -3.26 & -7.44 & $14.74 * *$ & $-13.83 * *$ & $-11.68 * *$ & $-12.45^{* *}$ & $20.63 * *$ & -5.24 & -6.89 & 0.37 & -3.49 & -1.02 \\
\hline 19. & ICPA 2156 x ICPL 88034 & $10.60 *$ & 5.81 & $18.95^{* * *}$ & $-10.67 * *$ & $-5.15^{* *}$ & $-5.98 * *$ & $19.37 * *$ & $-6.23 *$ & -4.81 & 2.61 & $13.64 *$ & $16.55^{* *}$ \\
\hline 20. & ICPA 2156 x ICPL 88039 & -2.77 & -6.98 & $21.58^{* *}$ & $-8.70 * *$ & $-12.56 * *$ & $-13.33 * *$ & $22.86^{* *}$ & -3.49 & -7.39 & -0.17 & -8.99 & -6.66 \\
\hline 21. & ICPA 2156 x ICPL 149 & 4.28 & -0.23 & $20.53^{* *}$ & $-9.49 * *$ & 0.00 & -0.87 & $21.59 * *$ & -4.49 & -7.96 & -0.78 & $-14.31^{*}$ & -12.12 \\
\hline 22. & ICPA 2156 x ICPL 161 & 6.47 & 1.86 & $19.47 * *$ & $-10.28 * *$ & 0.44 & -0.44 & $18.73 * *$ & $-6.73 *$ & -0.25 & 7.53 & 1.66 & 4.27 \\
\hline 23. & ICPA 2156 x ICPL 81-3 & $10.60^{*}$ & 5.81 & $20.00 * *$ & $-9.88 * *$ & -1.86 & -2.72 & $18.10^{* *}$ & $-7.23 * *$ & -4.72 & 2.71 & -1.66 & 0.85 \\
\hline 24. & ICPA 2156 x ICPL 89 & -1.80 & -6.05 & $11.58^{* *}$ & $-16.21 * *$ & 0.90 & 0.02 & $17.46^{* *}$ & $-7.73 * *$ & 0.63 & 8.47 & -3.16 & -0.68 \\
\hline 25. & ICPA $2156 \times$ ICPL 90048 & 0.63 & -3.72 & $11.58^{* * *}$ & $-16.21 * *$ & $-3.49 *$ & $-4.33 *$ & $17.14 * *$ & $-7.98 * *$ & 2.58 & 10.58 & $-14.81 *$ & $-12.63 *$ \\
\hline 26. & ICPA 2156 x ICPL 86022 & 4.52 & 0.00 & $10.00^{* *}$ & $-17.39 * *$ & 0.00 & -0.87 & $19.37 * *$ & $-6.23 *$ & -6.64 & 0.64 & $-13.64 *$ & -11.43 \\
\hline 27. & ICPA 2156 x ICPL 92047 & $9.04 *$ & 4.33 & $13.68 * *$ & $-14.62 * *$ & $-4.10^{*}$ & $-4.93 * *$ & $17.46^{* *}$ & $-7.73 * *$ & -7.48 & -0.27 & -3.00 & -0.51 \\
\hline & $\mathrm{SE}(\mathrm{d}) \pm$ & 5.48 & 5.48 & 1.91 & 1.91 & 1.67 & 1.67 & 3.61 & 3.61 & 0.75 & 0.75 & 1.21 & 1.21 \\
\hline & $\mathrm{CD}$ at $5 \%$ & 11.01 & 11.01 & 3.84 & 3.84 & 3.35 & 3.35 & 7.25 & 7.25 & 1.51 & 1.51 & 2.41 & 2.41 \\
\hline & $\mathrm{CD}$ at $1 \%$ & 14.68 & 14.68 & 5.12 & 5.12 & 4.45 & 4.45 & 9.65 & 9.65 & 2.02 & 2.02 & 3.22 & 3.22 \\
\hline
\end{tabular}

* - Significant at $5 \%$ level of significance, $* *$ - Significant at $1 \%$ level of significance

Table.3 Continued

\begin{tabular}{|c|c|c|c|c|c|c|c|c|c|c|c|}
\hline Sr. No & Crosses & \multicolumn{2}{|c|}{ No. of pods per plant } & \multicolumn{2}{|c|}{ No. of seeds per pod } & \multicolumn{2}{|c|}{100 seed wt. (g) } & \multicolumn{2}{|c|}{ Grain yield Per Plant (g) } & \multicolumn{2}{|c|}{ Harvest Index (\%) } \\
\hline & & SC 1 & SC 2 & SC 1 & SC 2 & SC 1 & SC 2 & SC 1 & SC 2 & SC 1 & SC 2 \\
\hline 1. & ICPA 2039 x ICPL 88034 & $56.84 * *$ & -5.23 & -5.00 & 3.64 & $-17.06^{* * *}$ & 2.10 & $27.29 * *$ & -6.43 & -3.20 & $-33.61 * *$ \\
\hline 2. & ICPA 2039 x ICPL 88039 & 1.50 & $-38.67 * *$ & -5.00 & 3.64 & $-18.09 * *$ & 0.84 & -9.62 & $-33.57 * *$ & 21.30 & $-16.81^{*}$ \\
\hline 3. & ICPA 2039 x ICPL 149 & $75.42 * *$ & $5.99 *$ & -6.67 & 1.82 & $-14.68 * *$ & 5.04 & $45.27 * *$ & 6.78 & $25.36^{*}$ & -14.02 \\
\hline 4. & ICPA 2039 x ICPL 161 & $126.33 * *$ & $36.76^{* *}$ & -5.83 & 2.73 & $-16.38 * *$ & 2.94 & $89.28 * *$ & $39.13 * *$ & $22.97 *$ & $-15.67 *$ \\
\hline 5. & ICPA 2039 x ICPL 81-3 & $25.42 * *$ & $-24.22 * *$ & -7.50 & 0.91 & $-13.65^{* *}$ & 6.30 & 4.58 & $-23.13 * *$ & 8.83 & $-25.36^{* *}$ \\
\hline 6. & ICPA 2039 x ICPL 89 & -5.66 & $-43.00 * *$ & -6.67 & 1.82 & $-20.48^{* *}$ & -2.10 & $-29.49 * *$ & $-48.17 * *$ & -9.11 & $-37.66^{* *}$ \\
\hline 7. & ICPA 2039 x ICPL 90048 & $77.67 * *$ & $7.36^{*}$ & -6.67 & 1.82 & -7.51 & $13.87 * *$ & $65.62 * *$ & $21.74 * *$ & 12.59 & $-22.79 * *$ \\
\hline 8. & ICPA 2039 x ICPL 86022 & -7.38 & $-44.03 * *$ & -5.00 & 3.64 & $-19.80 * *$ & -1.26 & $-32.10 * *$ & $-50.09 * *$ & $44.49^{* *}$ & -0.91 \\
\hline 9. & ICPA 2039 x ICPL 92047 & $46.81 * *$ & $-11.29 * *$ & -5.83 & 2.73 & $-24.57 * *$ & -7.14 & 9.78 & $-19.30 * *$ & -4.96 & $-34.82 * *$ \\
\hline 10. & ICPA 2089 x ICPL 88034 & $11.06^{*}$ & $-32.89 * *$ & $-12.50 * *$ & -4.55 & $-15.02 * *$ & 4.62 & -9.62 & $-33.57 * *$ & 13.63 & $-22.07 * *$ \\
\hline 11. & ICPA 2089 x ICPL 88039 & -9.14 & $-45.10 * *$ & 3.33 & $12.73 * *$ & $-17.06^{* *}$ & 2.10 & $-14.83 *$ & $-37.39 * *$ & $44.51 * *$ & -0.89 \\
\hline 12. & ICPA 2089 x ICPL 149 & $22.26 * *$ & $-26.13 * *$ & 0.00 & $9.09^{*}$ & $-24.23 * *$ & -6.72 & -10.57 & $-34.26^{* *}$ & 20.72 & $-17.21^{*}$ \\
\hline 13. & ICPA 2089 x ICPL 161 & 2.72 & $-37.94 * *$ & -5.83 & 2.73 & $-25.60 * *$ & -8.40 & $-19.08 * *$ & $-40.52 * *$ & $32.55 * *$ & -9.10 \\
\hline 14. & ICPA $2089 \times$ ICPL $81-3$ & $21.53^{* *}$ & $-26.57 * *$ & -5.00 & 3.64 & $-16.72 * *$ & 2.52 & 5.05 & $-22.78 * *$ & $25.16^{*}$ & -14.16 \\
\hline 15. & ICPA 2089 x ICPL 89 & $-17.13 * *$ & $-49.92 * *$ & -5.83 & 2.73 & $-14.68 * *$ & 5.04 & $-23.34 * *$ & $-43.65 * *$ & $55.48 * *$ & 6.63 \\
\hline 16. & ICPA 2089 x ICPL 90048 & $-30.33 * *$ & $-57.90 * *$ & -4.17 & 4.55 & $-8.19 *$ & $13.03^{* *}$ & $-29.26 * *$ & $-48.00 * *$ & -19.92 & $-45.08^{* * *}$ \\
\hline 17. & ICPA 2089 x ICPL 86022 & $-38.49 * *$ & $-62.83 * *$ & -7.50 & 0.91 & $-16.04 * *$ & 3.36 & $-47.00 * *$ & $-61.04 * *$ & $23.35^{*}$ & $-15.41^{*}$ \\
\hline
\end{tabular}

* - Significant at $5 \%$ level of significance, ** - Significant at $1 \%$ level of significance 
Table.3 Continued.....

\begin{tabular}{|c|c|c|c|c|c|c|c|c|c|c|c|}
\hline \multirow[t]{2}{*}{$\begin{array}{l}\text { Sr. } \\
\text { No }\end{array}$} & \multirow[t]{2}{*}{ Crosses } & \multicolumn{2}{|c|}{ No. of pods per plant } & \multicolumn{2}{|c|}{ No. of seeds per pod } & \multicolumn{2}{|c|}{100 seed wt. $(g)$} & \multicolumn{2}{|c|}{$\begin{array}{c}\text { Grain yield Per Plant } \\
\text { (g) }\end{array}$} & \multicolumn{2}{|c|}{$\begin{array}{c}\text { Harvest Index } \\
(\%)\end{array}$} \\
\hline & & SC 1 & SC 2 & SC 1 & SC 2 & SC 1 & SC 2 & SC 1 & SC 2 & SC 1 & SC 2 \\
\hline 18. & ICPA 2089 x ICPL 92047 & -1.83 & $-40.68 * *$ & -5.83 & 2.73 & $-12.97 * *$ & 7.14 & $-27.60 * *$ & $-46.78 * *$ & $34.86^{* *}$ & -7.51 \\
\hline 19. & ICPA 2156 x ICPL 88034 & $-20.10 * *$ & $-51.72 * *$ & -5.83 & 2.73 & $-15.36 * *$ & 4.20 & $-34.23 * *$ & $-51.65 * *$ & -0.89 & $-32.03 * *$ \\
\hline 20. & ICPA 2156 x ICPL 88039 & $-17.31 * *$ & $-50.04 * *$ & 0.00 & $9.09 *$ & $-7.85^{*}$ & $13.45 * *$ & $-19.08 * *$ & $-40.52 * *$ & $34.90 * *$ & -7.49 \\
\hline 21. & ICPA 2156 x ICPL 149 & 6.41 & $-35.70 * *$ & -6.67 & 1.82 & $-17.75 * *$ & 1.26 & $-19.08 * *$ & $-40.52 * *$ & $49.51 * *$ & 2.53 \\
\hline 22. & ICPA 2156 x ICPL 161 & $-15.25 * *$ & $-48.79 * *$ & -6.67 & 1.82 & $-21.50 * *$ & -3.36 & $-36.59 * *$ & $-53.39 * *$ & $43.35 * *$ & -1.69 \\
\hline 23. & ICPA 2156 x ICPL 81-3 & $-19.60 * *$ & $-51.42 * *$ & -5.00 & 3.64 & $-8.87 *$ & $12.18 *$ & $-25.28 * *$ & $-45.08 * *$ & $40.41 * *$ & -3.70 \\
\hline 24. & ICPA 2156 x ICPL 89 & $-25.08 * *$ & $-54.73 * *$ & -4.17 & 4.55 & -7.17 & $14.29 * *$ & $-33.28 * *$ & $-50.96 * *$ & -15.1 & $-41.77 * *$ \\
\hline 25. & ICPA 2156 x ICPL 90048 & $-19.45 * *$ & $-51.33 * *$ & 5.00 & $14.55 * *$ & $-7.85^{*}$ & $13.45 * *$ & $-24.29 * *$ & $-44.35 * *$ & $61.08 * *$ & 10.47 \\
\hline 26. & ICPA 2156 x ICPL 86022 & 1.63 & $-38.59 * *$ & $-10.00 *$ & -1.82 & $-16.72 * *$ & 2.52 & $-16.24^{*}$ & $-38.43 * *$ & 19.55 & $-18.01 *$ \\
\hline 27. & ICPA 2156 x ICPL 92047 & 2.33 & $-38.17 * *$ & $-10.83^{* *}$ & -2.73 & $-19.45 * *$ & -0.84 & $-16.86^{*}$ & $-38.89 * *$ & $51.45^{* *}$ & 3.87 \\
\hline & $\mathrm{SE}(\mathrm{d}) \pm$ & 9.57 & 9.57 & 0.16 & 0.16 & 0.37 & 0.37 & 4.52 & 4.52 & 3.14 & 3.14 \\
\hline & $\mathrm{CD}$ at $5 \%$ & 19.21 & 19.21 & 0.32 & 0.32 & 0.73 & 0.73 & 9.08 & 9.08 & 6.31 & 6.31 \\
\hline & $\mathrm{CD}$ at $1 \%$ & 25.60 & 25.60 & 0.43 & 0.43 & 0.98 & 0.98 & 12.1 & 12.1 & 8.41 & 8.41 \\
\hline
\end{tabular}

* - Significant at $5 \%$ level of significance, ** - Significant at $1 \%$ level of significance 
The range of standard heterosis was -12.50 to 5 and -4.55 to 14.55 percent over the check VL Arhar1 and ICPL 161 respectively. None of the crosses recorded significant positive heterosis over the check VL Arhar1. Over ICPL 161 maximum significant positive heterosis was registered by ICPA 2156 x ICPL 90048 (14.55\%) followed by ICPA 2089 x ICPL 88039 (12.73\%). These findings were in agreement with the findings of Patel and Tikka (2008), Sarode et al., (2009), Kumar et al., (2012), Pandey et al., (2013) and Sudhir et al., (2015).

The range of heterobeltiosis for the trait 100 seed weight is -18.64 to 16.24 percent. Maximum significant positive heterobeltiosis is exhibited by ICPA 2156 x ICPL 89 ($16.24 \%$ ) followed by ICPA 2156 x ICPL $81-3$ $(-14.10 \%)$. The range of standard heterosis for the trait 100 seed weight was -25.60 to -7.17 and -8.40 to 14.29 percent over the check VL Arhar1 and ICPL 161 respectively. None of the crosses showed significant positive heterosis over the check VL Arhar1. Six crosses recorded significant positive heterosis over the check ICPL161. Maximum significant positive heterosis is manifested by ICPA 2156 x ICPL 89 (14.29\%) followed by ICPA 2039 x ICPL 90048 (13.87\%) over the check ICPL 161. Heterosis with respect to 100 seed weight in positive and negative direction have also been reported by Wankhade et al., (2005), Baskaran and Muthiah (2006), Patel and Tikka (2008), Sarode et al., (2009), Kumar et al., (2012), Pandey et al., (2013) and Sudhir et al., (2015).

The ultimate goal of any breeding programme is to develop a high yielding hybrid. The high degree of heterosis for yield need not be due to the high heterosis in all yield contributing characters but may be of heterosis in one or two yield contributing characters even. A wide range of variation in the estimates of heterobeltiosis and standard heterosis in positive and negative direction was observed for grain yield per plant. For the trait, heterobeltiosis ranged from -55.53 to $83.25 \%$. Nine crosses manifested significant positive heterobeltiosis for this trait. Maximum significant positive heterobeltiosis is manifested by ICPA 2039 x ICPL 90048 (83.25\%) followed by ICPA 2156 x ICPL $86022(47.50 \%)$. The range of standard heterosis was -47.00 to 89.28 and -61.04 to 39.13 percent over the checks VL Arhar1 and ICPL 161 respectively. The cross ICPA 2039 $x$ ICPL $161(89.28 \%)$ exhibited significant positive heterosis over the check VL Arhar1 followed by ICPA 2039 x ICPL 90048 (65.62\%). Maximum significant positive heterosis is recorded by ICPA $2039 \mathrm{x}$ ICPL $161(39.13 \%)$ over the check ICPL 161 followed by ICPA 2039 x ICPL 90048 $(21.74 \%)$. These findings were in close agreement with the results of earlier workers Pandey and Singh (2002), Wankhade et al., (2005), Baskaran and Muthiah, (2006), Wanjari et al., (2007), Solanki et al., (2008), Patel and Tikka, (2008), Sarode et al., (2009), Singh and Singh, (2009), Dheva et al., (2009), Bharate et al., (2010), Chandrikala et al., (2010), Vaghela et al., (2011), Gupta et al., (2011), Kumar et al., (2012), Pandey et al., (2013) and Sudhir et al., (2015).

Harvest Index is one of the trait which indirectly influences the grain yield. The range of heterobeltiosis ranged from -40.52 to 36.99 percent for the trait harvest index. Maximum significant positive heterobeltiosis is recorded by ICPA $2089 \times$ ICPL 88039 (36.99\%) followed by ICPA $2156 \times$ ICPL $90048(23.36 \%)$. The range of standard heterosis was -19.92 to 61.08 and -45.08 to 10.47 percent over the checks VL Arhar1 and ICPL 161 respectively. Out of 27 crosses, 15 crosses exhibited significant positive heterosis over the check VL Arhar1. Maximum significant positive heterosis manifested by 
ICPA 2156 x ICPL $90048(61.08 \%)$ followed by ICPA 2089 x ICPL $89(55.48 \%)$ over the check VL Arhar1. None of the crosses showed significant positive heterosis over the check ICPL 161. The significant positive and negative heterosis for harvest index was also reported by Singh and Singh (2009), Dheva et al., (2009), Bharate et al., (2010), Gupta et al., (2011) and Pandey et al., (2013).

Ultimate aim of breeding is to gain the heterotic yield associated with the other heterotic characters. The estimates of heterosis showed that the crosses ICPA 2039 x ICPL161 and ICPA 2039 x ICPL 90048 had significant standard heterosis for grain yield per plant and some of its components. Exploitation of heterosis in short duration hybrids could pave a way to face the challenge of stagnant productivity in pigeonpea. These short duration hybrids also serve the purpose of getting fit into different agro-ecological niches.

\section{References}

Baskaran, K. and Muthiah, A.R. 2006. Variability studies in Pigeonpea (Cajanus cajan (L.) Millsp.) .Res.on Crops.7:249-252.

Bharate, B.S., Wadikar,P. B. and Pole, S. P.2010. Heterosis in pigeonpea. Ann. Plant Physiol. 24: 68-71.

Chandirakala, R., Subbaraman, N. and Abdul Hameed. 2010. Heterosis for yield in Pigeonpea (Cajanus cajan L. Millsp.). Electron. J. Plant Breed. 1(1): 205-208.

D.E.S, 2017. Directorate of Economics and Statistics, Department of Agriculture and Cooperation. Based on second estimate of 2016-17.

Dheva, N. G., Patil, A. N. and Wanjari, K. B. 2009. Heterosis in cytoplasmic male sterility based hybrids of pigeonpea. Int . J. Plant Sci. 4: 270-273.

FAOSTAT, 2015. http://faostat.fao.org/ foodstat/collections.

Fonesca, S. and Patterson, F. L. 1968. Hybrid vigour in a seven parental diallel cross in common winter wheat (Triticum aestivum L.). Crop Sci.8:85-88.

Gupta, D. K., Acharya, S. and Patel, J.B. 2011. Combining ability and heterosis studies in pigeonpea using $\mathrm{A}_{2}$ cytoplasm from Cajanus scarabaeoides as source of male sterility.J.Food Legumes.24(1): 58-64.

Kumar, C.V.S., Sreelakshmi, C.H. and Shivani, D. (2012). Gene effects, heterosis and inbreeding depression in pigeonpea (Cajanus cajan L.). Electron. J. Plant Breed. 3:682-685.

Pandey, N. and Singh, N.B. 2002. Hybrid vigour and combining ability in long duration pigeonpea (Cajanus cajan (L.) Millsp) hybrids involving male sterile lines. Indian J.Genet.Plant Breed.62(3): 221-225.

Pandey, P., Rajesh, K., Pandey, V. R, Jaiswal, K. K. and Tripathi, M., 2013. Studies on heterosis for yield and its component traits on CGMS based pigeonpea [Cajanus cajan (L.) Millsp.] hybrids. Int. J. Agric. Res.8: 158-171.

Patel, M. P. and Tikka, S. B. S., 2008. Heterosis for yield and yield components in pigeonpea. J. Food Legumes.2: 65-66.

Sarode, S.B., Singh, M.N. and Singh, U.P. 2009. Heterosis in long duration pigeonpea (Cajanus cajan (L.) Millsp.). Int. J.Agric.Sci.4(1): 106-108.

Saxena, K.B., and Nadarajan N.2010.Prospects of Pigeonpea hybrids in Indian Agriculture. Electron. J. Plant Breed. 1:1107-1117.

Saxena, K.B., Chauhan, V.S., Johansen, C. and Singh, L.,1992. Recent Developments in Hybrid Pigeonpea Research. Proceedings of Workshop on 'New Frontiers in Pulses Research and Development.' November10 to 12, 1989, 
Kanpur, India, pp:58-69.

Shoba, D. and Balan, A. 2010. Heterosis in CMS/GMS based Pigeonpea [Cajanus cajan (L.) Millsp.] hybrids. Agric. Sci. Digest. 30 (1): $32-36$.

Singh, O. and Singh, M.N. 2009. Combining ability analysis in pigeonpea. J. Food Legumes. 22(1): 30-33.

Solanki, S. D., Jaimini, S.N., Patel. J. B. and Chauhan. R. M., 2008. Heterosis study in interspecific cross of pigeonpea [Cajanus scarabaeoides $x$ Cajanus cajan (L.) Millsp.]. Biosci.Rep.6: 95-98.

Sudhir, K., Debnath, M. K., Sameer Kumar, C. V., Singh, P. K. and Sultana. R. 2015. Study of heterosis and pollen fertility in CGMS based pigeonpea [Cajanus cajan (L.) Millspaugh] hybrids. Res. Environ. Life Sci. 9(1) 107-110.

Vaghela, K. O., Desai, R. T., Nizama, J. R., Patel, J. D., and Kodappully, V. C., 2011. Heterosis study for yield and yield components in pigeonpea (Cajanus cajan (L.) Millsp.]. Res. on Crops, 12: 192-194.

Wanjari, K. B., Bhongale, S.A. and Sable, N.H. 2007. Evaluation of heterosis in CMS based hybrids in pigeonpea. J.Food Legumes. 20(1): 107-108.

Wankhade, R. R., Wanjari, K. B., Kadam, G. M. and Jadhav, B. P., 2005. Heterosis for yield and yield components in pigeonpea involving male sterile lines. Indian J.Pulses Res.18: 141-143.

\section{How to cite this article:}

Srivarsha, J., J.E. Jahagirdar, C.V. Sameer Kumar, A.J. Hingane, D.K. Patil, V.K. Gite, H.B. Shruthi and Bhosle, T.M. 2017. Study of CGMS based Short Duration Hybrids of Pigeonpea [Cajanus cajan (L.) Millsp.] in Terms of Heterosis. Int.J.Curr.Microbiol.App.Sci. 6(11): 682692. doi: https://doi.org/10.20546/ijcmas.2017.611.081 